\title{
ESTUDIO ANÁTOMO-HISTOPATOLÓGICO INTESTINAL EN EL TRATAMIENTO CON METRONIDAZOL EN ENTERITIS AGUDA INDUCIDA POR INDOMETACINA EN RATAS ALBINAS
}

\author{
Beatriz Moscol A. ${ }^{1}$, Alfonso Chavera C. ${ }^{2,3}$ y Pedro Angulo H. ${ }^{4}$
}

Abstract

\begin{abstract}
The anatomo histopathological characteristics of the intestine in rats with acute enteritis induced by injection of one dose of indomethacine and treated with metronizadole were evaluated. Male albino Holtzman rats $(n=50)$ were used. They were 4 months old on average and $250-275 \mathrm{~g}$ of body weight. Animals were equally distributed in five groups: Control (Group 1), subcutaneously injected with indomethacine (15 mg/kg) (Group 2), and injected with indomethacine plus 1,2 or 3 doses of metronizadole $(50 \mathrm{mg} / \mathrm{kg}$ ) (groups 3,4 and 5, respectively). The first dose of metronizadole was orally administered 50 minutes before the indomethacine and subsequent doses were every 5 hours. The observation of lesions was done 48 hours later. Lesions were located in the mesenteric side of the medium and distal jejune. Macroscopic lesions were characterized by thickness and dilatation of the intestinal wall, loss of the mucosa, hyperemia, and the absence of lineal ulcers longer than $10 \mathrm{~cm}$. No statistical differences were found between groups treated with metronizadole ( $p>0.05)$; however, differences between these groups with the indomethacine group $(\mathrm{p}=0.021)$ were found. Microscopic lesions were characterized by erosion of the mucosa, presence of fibrin and mienteric plexus degeneration. No differences between the metronizadole treated groups $(\mathrm{p}>0.05)$ were found.
\end{abstract}

Key words: metronizadole, indomethacine, inflammation, rat

\section{Resumen}

\begin{abstract}
Se evaluaron las características anatómicas e histopatológicas del intestino de ratas con enteritis aguda inducida con una dosis de indometacina y tratadas con metronidazol. Se usaron 50 ratas albinas machos de la raza Holtzman, de 4 meses de edad promedio y de 250-275 g de peso vivo. Los animales se distribuyeron por igual en 5 grupos. El Lote 1 fue el lote control, el Lote 2 recibió únicamente la indometacina $(15 \mathrm{mg} / \mathrm{kg}$ p.v. por vía sucutánea), y los lotes 3, 4 y 5 recibieron la indometacina, además de una, dos y tres dosis de metronizadol (50 mg/kg p.v. por vía oral), respectivamente. La primera dosis de metronizadol se administró 50 minutos previos a la indometacina, y las demás dosis con intervalos de 5 horas. La observación de las lesiones se realizó a las 48 horas. Todas las lesiones fueron halladas en el lado mesentérico del yeyuno medio y distal. Las lesiones macroscópicas se caracterizaron por engrosamiento y dilatación de la pared intestinal, pérdida de la mucosa e hiperemia, sin presencia de úlceras lineales mayores de $10 \mathrm{~cm}$. No
\end{abstract}

\author{
${ }^{1}$ Práctica privada \\ ${ }^{2}$ Laboratorio de Histología, Embriología y Patología Veterinaria, FMV-UNMSM \\ ${ }^{3}$ E-mail: achavera03@yahoo.com \\ ${ }^{4}$ Laboratorio de Farmacología, Toxicología y Terapéutica Veterinaria, FMV-UNMSM
}


se encontraron diferencias en las lesiones macroscópicas entre los grupos tratados con metronizadol ( $p>0.05$ ), pero hubo diferencia entre estos con el grupo de indometacina sola $(\mathrm{p}=0.021)$. Las lesiones microscópicas se caracterizaron por erosión de la mucosa, presencia de fibrina y degeneración del plexo mioentérico. No se encontró diferencia estadística entre los grupos tratados con metronizadol ( $p>0.05)$.

Palabras clave: metronizadol, indometacina, inflamación, rata

\section{INTRODUCCIón}

Las patologías gastrointestinales, y en especial las enteritis, tienen un gran impacto económico en la salud y producción animal en el país. Los diversos cuadros entéricos son frecuentes y siempre perjudiciales en el crecimiento y desarrollo de muchas especies. Una enfermedad entérica causada por Escherichia coli ocasiona el $50 \%$ de pérdidas de lechones antes del destete y reducciones en el ritmo de crecimiento e indices de conversión de 30 a 50\% (Camacho, 1997). Por otro lado, se reconoce que la enterotoxemia constituye la principal causa de pérdidas en crías de alpacas y llamas llegando hasta el 40 a $50 \%$ durante la presentación de brotes (Ramírez, 1990).

La presentación de una enfermedad entérica está determinada por circunstancias tales como la ausencia de inmunidad, alteración de la actividad del intestino (estrés, tipo de alimento, sobrealimentación, etc.), procesos infecciosos (bacterias, virus, parásitos) o tóxicos (toxinas bacterianas, micotoxinas, quimicos tóxicos, etc.) (Camacho, 1997).

Muchas investigaciones evidencian que los radicales libres y los oxidantes tienen un rol importante en el daño a nivel celular, y en consecuencia dan lugar a lesiones tisulares como las úlceras. Este proceso oxidativo puede agravarse, y de hecho asi sucede, por la disminución de antioxidantes fisiológicos en el proceso inflamatorio (Gross et al., 1994). El metronidazol ha demostrado ser un barredor de radicales libres, reduciendo las especies reactivas derivadas del oxígeno generadas por los neutrófilos en el sitio de la inflamación (Davies et al., 1993; Milks et al., 1986).
En ratas albinas libres de gérmenes o en aquellas con tratamiento a base de antibióticos se ha observado que quedan protegidas de perforaciones ileales retardadas inducidas por indometacina. Esta evidencia sugiere que la patogénesis del daño intestinal inducido por los AINES tiene más de una fase: inicialmente se tiene un daño superficial en la mucosa, probablemente relacionado a los efectos de la droga en sí (por ejemplo, depleción local de prostaglandina), seguido de un daño de la mucosa por parte de los contenidos luminares perjudiciales, incluyendo la flora bacteriana, ocasionando una inflamación más severa y a veces la perforación (Davies et al., 1993). Por otro lado, con la administración de metronidazol se puede observar la disminución de la permeabilidad intestinal inducida por la indometacina (Davies y Jamali, 1997).

La motivación del presente trabajo fue prevenir el proceso inflamatorio intestinal con la utilización de productos naturales con actividad antioxidante y antiinflamatoria, pero para ello se requiere de modelos experimentales en animales con productos de farmacología conocida. Para esto, se utilizó la indometacina a fin de provocar una enteritis aguda en ratas albinas Holtzman, basándose en el método experimental propuesto por Yamada et al. (1993) y modificado por Ramos (2003), en el cual se hace una evaluación anátomohistopatológica del grado de acción del metronidazol en el intestino delgado en el momento de mayor daño.

\section{Materiales y Métodos}

El estudio se realizó entre los meses de mayo y setiembre del 2002, en el Laboratorio 
de Farmacología, Toxicología y Terapéutica Veterinaria y en el Laboratorio de Histopatología Veterinaria de la Facultad de Medicina Veterinaria de la Universidad Nacional Mayor de San Marcos.

\section{Animales y manejo}

Se utilizaron 50 ratas albinas macho de la raza Holtzman, de 4 meses de edad y 250275 gramos de peso vivo. Los animales fueron acondicionados en el laboratorio durante 20 días como periodo de acostumbramiento, donde se mantuvieron en lotes de 10 animales en jaulas metálicas, recibiendo agua y alimento ad libitum (30 g por animal de alimento comercial) con cambio diario de cama (viruta) .

\section{Tratamientos}

Se utilizaron 5 lotes de 10 animales cada uno.

- Lote 1: Sin medicación.

- Lote 2: Inyección subcutánea de indometacina.

- Lote 3: Una dosis de metronidazol e indometacina a los 50 minutos.

- Lote 4: Dos dosis de metronidazol a intervalos de 5 horas e indometacina a los 50 minutos de la primera dosis.

- Lote 5: Tres dosis de metronidazol a intervalos de 5 horas e indometacina a los 50 minutos de la primera dosis.

La indometacina se inyectó vía subcutánea (disuelta en bicarbonato de sodio al 5\%) en dosis de $15 \mathrm{mg} / \mathrm{kg}$ p.v. El metronidazol se dio por vía oral en dosis de $50 \mathrm{mg} / \mathrm{kg}$ p.v. $(1.25 \%)$.

Al segundo día de iniciado el experimento, se anestesiaron a los animales con pentobarbital sódico vía intraperitoneal y se procedió a una laparotomía exploratoria. Se tomó aproximadamente $10 \mathrm{~cm}$ de la parte media del intestino delgado para la evaluación de las lesiones. Los animales se eutanasiaron por shock hipovolémico, luego de tomada la muestra.

\section{Evaluación del daño intestinal}

Para la determinación del daño tisular y la inflamación del intestino delgado, la muestra colectada fue abierta longitudinalmente por el lado antimesentérico, seleccionando la sección correspondiente al yeyuno ileon (Yamada et al.,1993). Las lesiones inflamatorias se evaluaron utilizando la clasificación de Slauson y Cooper (1990).

El grado de lesión se evidenció por el tamaño e intensidad de las lesiones intestinales de cada tratamiento y en base a esto se empleó la prueba no paramétrica de Kruskall Wallis.

\section{Resultados}

En el presente trabajo se administró metronidazol como preventivo y tratamiento en una enteritis aguda inducida por una sola aplicación de indometacina, con la finalidad de observar su grado de protección. Al observar los hallazgos microscópicos, se nota que las lesiones típicas de inflamación están ausentes en la mayoría de los animales, siendo el engrosamiento de la pared intestinal e hiperemia los más evidentes en los lotes 3 y 5 , respectivamente; asimismo, se aprecia que estas lesiones representan un tercio del total de las lesiones (Cuadro 1). Además, es notable la ausencia de exudado mucoso en los tres grupos de animales tratados con metronidazol así como los pocos casos de úlceras (Cuadro 1).

Los parámetros inflamatorios a nivel tisular se muestran en el Cuadro 2. Los daños tisulares se pudieron reproducir en el Lote 2 (Fig. 1). Se pudo observar la mucosa erosionada y el corion expuesto como lesión predominante en ratas de los lotes 4 y 5 (Fig. 2). Así mismo, se observó lesiones acompañadas de fibrina en un tercio de los casos, presencia de neutrófilos y moderado número de linfocitos, con necrosis, vacuolización y degeneración del plexo mioentérico (Fig. 3). 
Cuadro 1. Hallazgos macroscópicos de lesiones intestinales (yeyuno) en 10 ratas albinas por grupo experimental con enteritis inducida por indometacina y tratadas con metronidazol

\begin{tabular}{|c|c|c|c|c|c|c|}
\hline \multirow{2}{*}{ Observaciones } & \multicolumn{5}{|c|}{ Animales afectados } & \multirow{2}{*}{$\begin{array}{l}\text { Total de } \\
\text { Afectados }\end{array}$} \\
\hline & Lote $1^{2}$ & Lote 2 & Lote 3 & Lote 4 & Lote 5 & \\
\hline $\begin{array}{l}\text { Engrosamiento de la } \\
\text { pared intestinal }\end{array}$ & 0 & 6 & 2 & 2 & 5 & 9 \\
\hline $\begin{array}{l}\text { Dilatación de la pared } \\
\text { intestinal }\end{array}$ & 0 & 7 & 3 & 3 & 4 & 10 \\
\hline Mucosa barrida & 0 & 1 & 1 & 2 & 1 & 4 \\
\hline Hiperemia & 0 & 6 & 4 & 1 & 5 & 10 \\
\hline $\begin{array}{l}\text { Exudado mucoso } \\
\text { - amarillento } \\
\text { - } \text { rojizo }\end{array}$ & $\begin{array}{l}0 \\
0\end{array}$ & $\begin{array}{l}6 \\
4\end{array}$ & $\begin{array}{l}1 \\
0\end{array}$ & $\begin{array}{l}0 \\
0\end{array}$ & $\begin{array}{l}1 \\
0\end{array}$ & $\begin{array}{l}2 \\
0\end{array}$ \\
\hline $\begin{array}{l}\text { Úlceras } \\
\text { - únicas y múltiples } \\
\text { - lineales } \\
\text { - ausencia de úlceras }\end{array}$ & $\begin{array}{c}0 \\
0 \\
10\end{array}$ & $\begin{array}{l}8 \\
6 \\
1\end{array}$ & $\begin{array}{l}2 \\
1 \\
8\end{array}$ & $\begin{array}{l}3 \\
1 \\
7\end{array}$ & $\begin{array}{l}2 \\
1 \\
8\end{array}$ & $\begin{array}{c}7 \\
3 \\
23\end{array}$ \\
\hline
\end{tabular}

${ }^{1}$ Sobre un total de 30 (lotes 3,4 y 5 )

${ }^{2}$ Lote 1: Control; 2: Indometacina (I); $3: 1+$ una dosis de metronidazol (M); $4: 1+2$ dosis de $M ; 5: 1+3$ dosis de $\mathrm{M}$

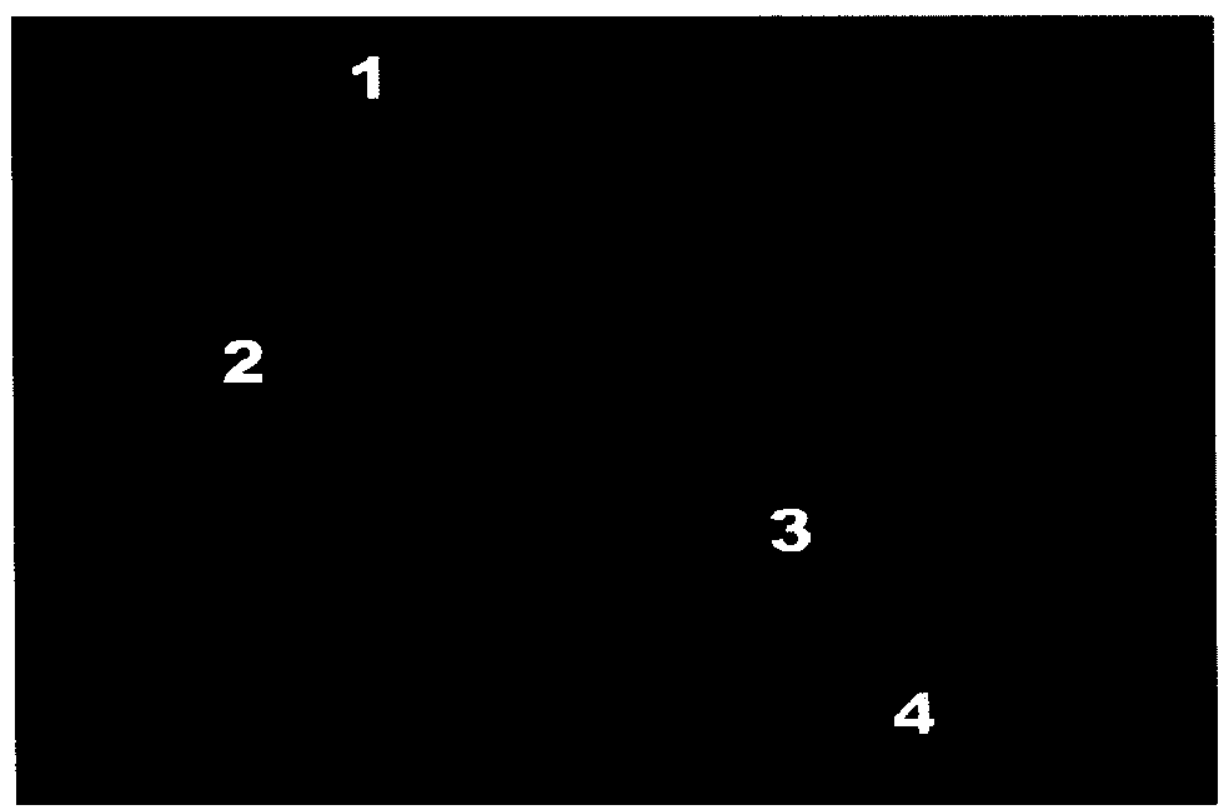

Figura 1. Intestino delgado (Lote 2, inyección de indometacina), sección yeyuno-ileon (corte transversal). Presencia de zona uicerada tapizada con exudado fibrinoso (1) y bacterias (2). Se observa además neutrófilos y linfocitos (3), acompañado de severa necrosis en la capa muscular (4). $H-E, 100 x$. 


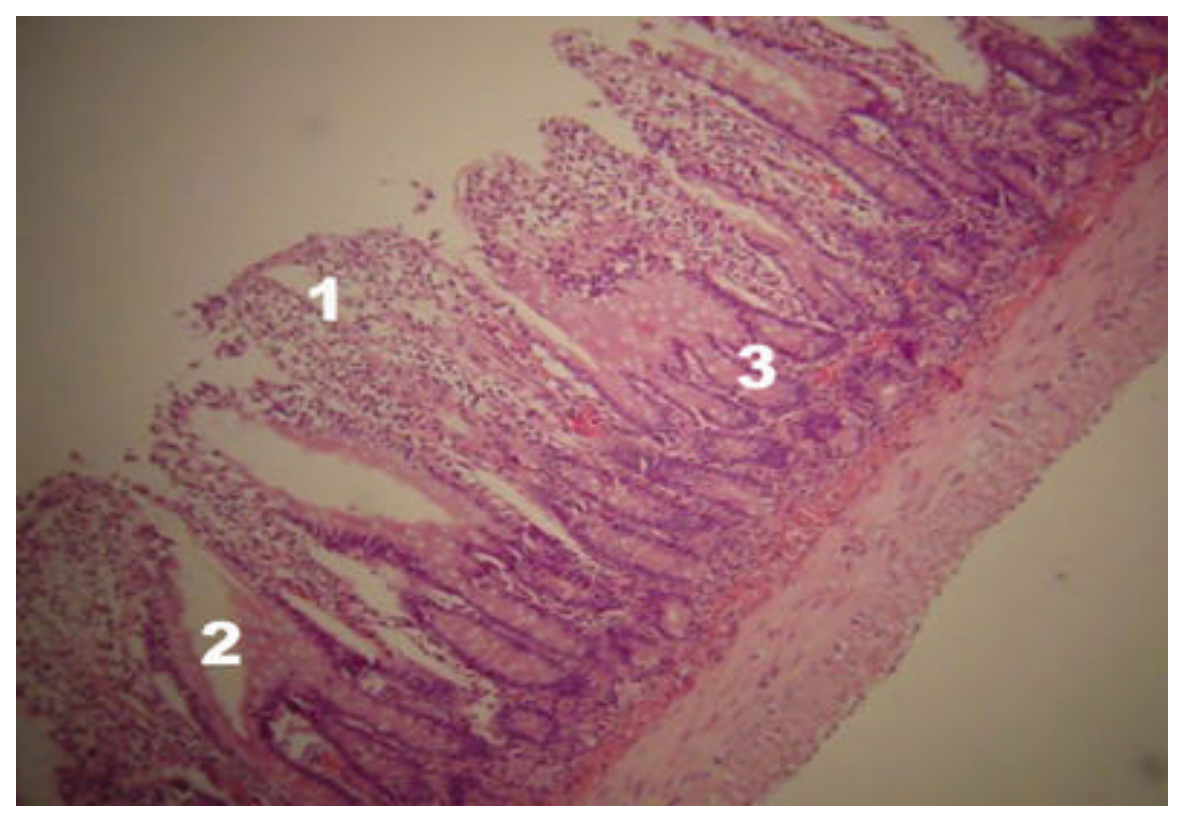

Figura 2. Intestino delgado (Lote 3, Inyección de indometacina con una dosis de metronidazol). Se observa la erosión de la mucosa (1), corion expuesto (2) y submucosa con pérdida inicial de su arquitectura (3). H-E, 100x

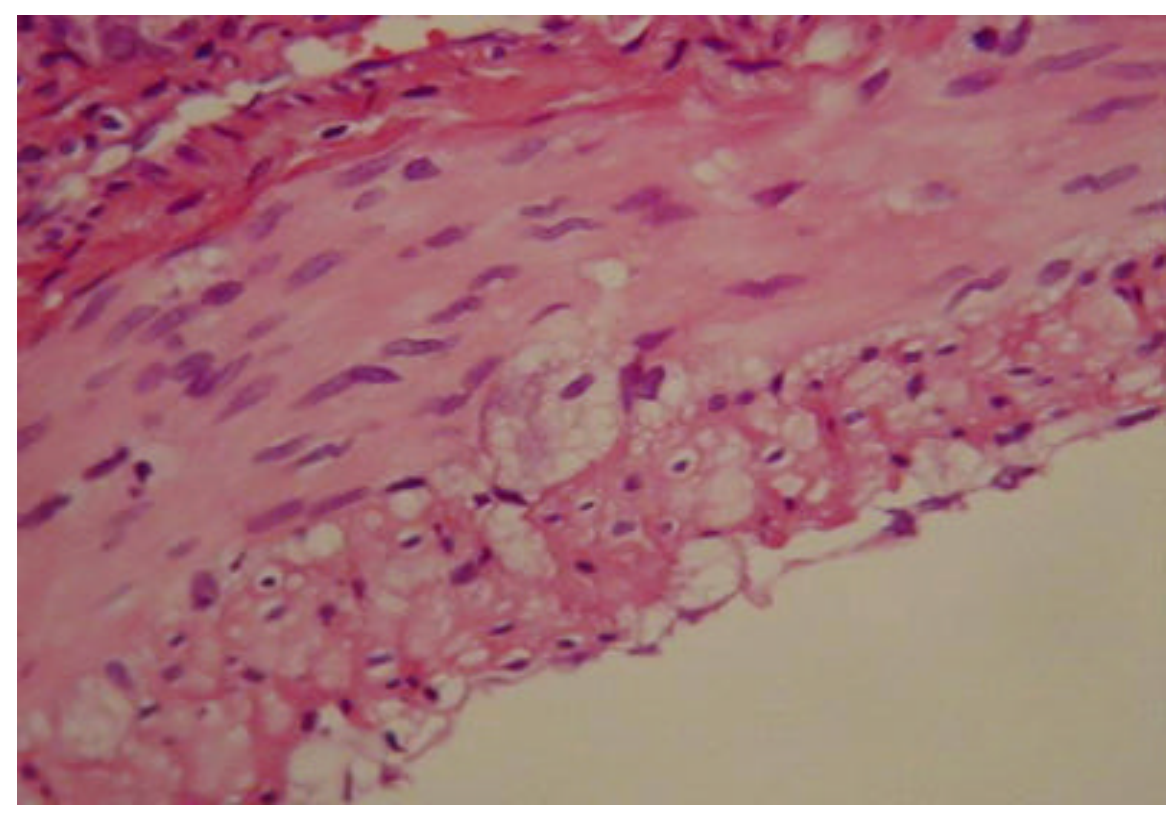

Figura 3. Intestino delgado (Lote 3, Indometacina con una dosis de metronidazol). Se observa vacuolización y degeneración del plexo mioentérico y de la capa muscular. H-E, 400x 
Cuadro 2. Hallazgos microscópicos de lesiones intestinales (yeyuno) en 10 ratas albinas por grupo experimental con enteritis inducida por indometacina y tratadas con metronidazol

\begin{tabular}{lcccccc}
\hline \multirow{2}{*}{ Parámetros inflamatorios } & \multicolumn{5}{c}{ Animales afectados } & Total de \\
\cline { 2 - 6 } & Lote $1^{2}$ & Lote 2 & Lote 3 & Lote 4 & Lote 5 & Afectados \\
\hline Erosión de la mucosa & 0 & 8 & 5 & 10 & 10 & 25 \\
Corion expuesto & 0 & 3 & 0 & 7 & 7 & 14 \\
Presencia de fibrina & 0 & 5 & 4 & 2 & 5 & 11 \\
Presencia de bacterias & & & & & & \\
$\quad$ - Leve & 0 & 0 & 0 & 1 & 1 & 2 \\
$\quad$ - Moderado & 0 & 3 & 4 & 1 & 2 & 7 \\
$\quad-\quad$ Severo & 0 & 6 & 0 & 0 & 0 & 0
\end{tabular}

Exudado neutrofilico
- Leve
- Moderado
- Severo

Exudado linfocitario

$0 \quad 9$

$0 \quad 0$

$0 \quad 0$

0

1
4
0

1
3
1

$\begin{array}{cc}2 & 4 \\ 4 & 11 \\ 1 & 2\end{array}$

Necrosis de la mucosa

\section{- Leve \\ - Moderado}

- Severo

Necrosis de la muscular $y$ vacuolización

- Leve

- Moderado

- Severo

Vacuolización y degeneración de plexo mioentérico

Presencia de fibroblastos con neoformación de vasos
- Leve

- Moderado

- Severo

Perforaciones con seropaniculitis

$\begin{array}{cccccc}0 & 9 & 2 & 3 & 5 & 10 \\ 0 & 0 & 8 & 5 & 5 & 18 \\ 0 & 0 & 0 & 1 & 0 & 1 \\ 0 & 9 & 5 & 5 & 6 & 16\end{array}$

4
2
1
0
18
1
16

\footnotetext{
${ }_{2}^{1}$ Sobre un total de 30 (lotes 3,4 y 5 )

${ }^{2}$ Lote 1: Control; 2: Indometacina (1); $3: 1+$ una dosis de metronidazol (M); $4: 1+2$ dosis de $M ; 5: 1+$ 3 dosis de $M$
} 
Cuadro 3. Hallazgos macroscópicos de lesiones vasculares intestinales (yeyuno) en 10 ratas albinas por grupo experimental con enteritis inducida por indometacina y tratadas con metronidazol

\begin{tabular}{|c|c|c|c|c|c|c|}
\hline \multirow{2}{*}{ Parámetros vasculares } & \multicolumn{5}{|c|}{ Animales afectados } & \multirow{2}{*}{$\begin{array}{l}\text { Total de } \\
\text { afectados }\end{array}$} \\
\hline & Lote $1^{2}$ & Lote 2 & Lote 3 & Lote 4 & Lote 5 & \\
\hline $\begin{array}{l}\text { Arteria con endotelio destruido } \\
\text { y cepa muscular vacuolizada }\end{array}$ & 0 & 9 & 6 & 7 & 7 & 20 \\
\hline Vena con endotelio destruido & 0 & 9 & 7 & 7 & 7 & 21 \\
\hline Arteriola con microtrombo & 0 & 9 & 5 & 4 & 6 & 15 \\
\hline $\begin{array}{c}\text { Edema en subserosa } \\
\text { - Leve } \\
\text { - Moderado } \\
\text { - Severo }\end{array}$ & $\begin{array}{l}0 \\
0 \\
0\end{array}$ & $\begin{array}{l}0 \\
0 \\
9\end{array}$ & $\begin{array}{l}0 \\
5 \\
0\end{array}$ & $\begin{array}{l}0 \\
5 \\
0\end{array}$ & $\begin{array}{l}0 \\
5 \\
0\end{array}$ & $\begin{array}{c}0 \\
15 \\
0\end{array}$ \\
\hline
\end{tabular}

Cuadro 4. Grados de lesión macroscópica del intestino afectado (yeyuno) en 10 ratas albinas por grupo experimental con enteritis inducida por indometacina y tratadas con metronidazol, según la escala de Yamada et al. (1993)

\begin{tabular}{lcccccc}
\hline & \multicolumn{5}{c}{ Animales evaluados } & \multicolumn{1}{c}{$\begin{array}{c}\text { Total de } \\
\text { Grado }\end{array}$} \\
\cline { 2 - 6 } & Lote $1^{2}$ & Lote 2 & Lote 3 & Lote 4 & Lote 5 & afectos $^{1}$ \\
\hline Cero & 10 & 1 & 2 & 1 & 3 & 6 \\
Uno & 0 & 1 & 4 & 1 & 0 & 5 \\
Dos & 0 & 0 & 1 & 4 & 0 & 5 \\
Tres & 0 & 0 & 1 & 1 & 5 & 7 \\
Cuatro & 0 & 2 & 2 & 3 & 2 & 7 \\
Cinco & 0 & 6 & 0 & 0 & 0 & 0 \\
\hline
\end{tabular}

${ }^{1}$ Sobre un total de 30 (lotes 3,4 y 5 )

${ }^{2}$ Lote 1: Control; 2: Indometacina (I); $3:$ I + una dosis de metronidazol (M); $4: I+2$ dosis de $M ; 5: I+3$ dosis de $\mathrm{M}$

${ }^{3}$ Grado 0: sin cambio en la mucosa y serosa Grado 1:hiperemia y/o sangrado petequial Grado 2:erosión de la mucosa o ulceración

Grado 3: sólo erosión de la mucosa o ulceración con lesiones hiperémicas, adhesivas o hemorrágicas en la serosa y mesenterio

Grado 4:múltiples erosiones o ulceraciones $(<10 \mathrm{~cm})$

Grado 5:múltiples erosiones o ulceraciones $(>10 \mathrm{~cm})$ 
Cuadro 5. Grados de lesión microscópica del intestino afectado (yeyuno) en 10 ratas albinas por grupo experimental con enteritis inducida por indometacina y tratadas con metronidazol, usando la clasificación de Slauson y Cooper (1990)

\begin{tabular}{|c|c|c|c|c|c|c|}
\hline \multirow{2}{*}{ Grado $^{3}$} & \multicolumn{5}{|c|}{ Animales evaluados } & \multirow{2}{*}{$\begin{array}{c}\text { Total de } \\
\text { Afectados }\end{array}$} \\
\hline & Lote $1^{2}$ & Lote 2 & Lote 3 & Lote 4 & Lote 5 & \\
\hline Sin lesión & 10 & 1 & 1 & 1 & 1 & 3 \\
\hline Leve & 0 & 0 & 4 & 1 & 0 & 5 \\
\hline Moderado & 0 & 2 & 1 & 3 & 5 & 9 \\
\hline Severo & 0 & 7 & 4 & 5 & 4 & 13 \\
\hline
\end{tabular}

${ }^{1}$ Sobre un total de 30 (lotes 3,4 y 5 )

${ }^{2}$ Lote 1: Control; 2: Indometacina (I); $3: 1+$ una dosis de metronidazol (M); $4: I+2$ dosis de $M ; 5: I+3$ dosis de $M$

${ }^{3}$ Grado leve: incluye escasa destrucción de tejido, escasas evidencias de daño vascular (hiperemia y edema) y poca exudación.

Grado moderado: la reacción usualmente comprende una parte de tejido dañado y hallazgos visibles de reacción inflamatoria a la injuria, manifestada por la acumulación de leucocitos y fenómenos vasculares.

Grado severo: comprende la extensión de la reacción moderada, en la cual se observa considerables daños del tejido en una gran extensión y abundante exudación.

La presencia de linfocitos y neutrófilos en grado moderado indica una resolución de las lesiones, posiblemente debido a la mínima presencia de bacterias (acción antibiótica) o a la posible inhibición de adherencia leucocitaria provocada por el metronidazol o por medio de la posible formación de óxido nítrico a partir de éste.

Así mismo, la mayor cantidad de casos con lesiones moderadas en los animales del Lote 3 se debió a la mayor cantidad de bacterias existentes, puesto que en ese caso sólo se usó una dosis de metronidazol. Por otro lado, los casos de perforaciones con seropaniculitis fueron mínimas en los lotes 4 y 5 (Cuadro 2).

Los daños vasculares se dieron en todos los grupos. La destrucción endotelial de las arterias y venas conjuntamente con el edema moderado en la subserosa se observó en la mayoría de los animales tratados con metrozinadol (Cuadro 3).

Entre las lesiones macroscópicas, según la clasificación de Yamada et al. (1993), se pudo apreciar la ausencia de erosiones múltiples o ulceraciones mayores de $10 \mathrm{~cm}$ (grado 5) (Cuadro 4) en todos los tratamientos.

Los hallazgos microscópicos, según la clasificación de Slauson y Cooper (1990), muestran que entre cuatro y cinco animales por lote presentaron lesiones severas, lo cual representa menos del $50 \%$ del total de animales tratados con metromidazol; y por lo menos, uno no presentó algún tipo de lesión. Además, cabe mencionar que los animales del Lote 3 ( 1 dosis) tuvieron más lesiones leves que los otros que recibieron dosis adicionales de metronidazol (Cuadro 5).

A] analizar los datos por medio de la prueba no paramétrica de Kruskall Wallis se pudo observar que el grado de acción del metronizadol (tanto en los hallazgos macroscópicos como en los microscópicos) no tuvieron mayor diferencia estadística entre los tres lotes tratados. Sin embargo, existió diferencia significativa estadística entre los lotes tratados con metronidazol y aquel que no lo tuvo (Lote 2$)(\mathrm{p}=0.021)$, pero sólo en lo que se refiere a lesiones macroscópicas, mientras que en las lesiones microscópicas no 
existe esta diferencia, aunque sí una tendencia a que los animales con una dosis de metronizadol presenten lesiones microscópicas menores.

\section{Discusión}

Ramos (2003) propuso un modelo de enteritis aguda inducida por indometacina, con una dosis similar a la del presente estudio, en ratas albinas de raza Holtzman machos, logrando lesiones en todos los animales, comprobando que las lesiones quedaban limitadas al intestino delgado medio. Similarmente, se pudo reproducir lesiones en el lado mesentérico del yeyuno, reportadas en otros trabajos, y más específicamente en los lugares irrigados por los capilares (Anthony et al., 1997). En el presente estudio se pudo reproducir las mismas lesiones, todas ellas en el lado mesentérico del yeyuno (Fig. 1).

Las lesiones intestinales halladas en el presente estudio se encontraron en el yeyuno medio y terminal. Wax et al. (1970), por otro lado, demostraron mediante estudios toxicológicos en animales que las lesiones aparecen típicamente en el yeyuno terminal y el ileon, ya que es el sitio de absorción activa de la bilis.

Se pudo comprobar el efecto protector del metronidazol, pero aún no se sabe cuales son los mecanismos de protección. Yamada et al. (1993) sugieren que es debido a su acción antimicrobiana. Por otro lado, Davies y Jamali (1997) demuestran que el metronidazol protege contra el aumento de la permeabilidad intestinal inducida por la indometacina en una etapa temprana (12 horas antes de la inducción) y concluyen que el metronidazol es dependiente de la dosis y que en dosis elevadas, interfiere cuando la participación de las bacterias luminales es poco evidente.

Evans y Whittle (2001), al administrar indometacina en ratas albinas, incrementaron el nivel vascular de la albúmina en el yeyuno, asociado con la inducción del óxido nítrico sintetasa inducible. Al tener estas evidencias, administró metronidazol (200mg/kg/día) en forma preventiva, por lo que atribuye el efecto protector del metronidazol a la reducción del daño microvascular y a prevenir la expresión de la actividad del óxido nítrico sintetasa inducible (iNOS). Tal como se puede observar en el Cuadro 3, los daños vasculares son elevados en el Lote 2, en los cuales solo se empleó indometacina; mientras que en los lotes 3 , 4 y 5 tuvieron menos animales afectados a ese nivel.

Los resultados de este trabajo confirman estudios previos en donde el metronidazol fue asociado con la reducción significativa en el daño intestinal macroscópico inducido por indometacina. Además, el metronidazol previno los cambios en la permeabilidad intestinal inducida por AINES 48 horas después de la administración de indometacina, donde se ha reportado en estudios anteriores que es el momento de mayor injuria. Al encontrar cierta protección en el Lote 3 , se puede sugerir que el metronidazol interviene en una fase temprana del daño intestinal con una pequeña o ninguna influencia de los agentes ofensivos luminales.

\section{Conclusiones}

- El metronidazol protege el intestino delgado de la inflamación aguda provocada por una sola dosis de indometacina.

- El efecto protector del metronidazol se atribuye no sólo al aspecto antibacteriano de la droga, sino a otros que todavía están en estudio.

- No hubo diferencia estadística entre los 3 grupos tratados con metronidazol, pero sí hubo diferencia significativa entre estos con el lote control de indometacina sola, en lo que se refiere a lesiones macroscópicas. 


\section{LTTERATURA CITADA}

1. Anthony, A.; R.E. Pounder; P. Dhillon; A.J. Wakefield. 1997. Vascular anatomy defines sites of indomethacin induced jejunal ulceration along the mesenteric margin. Gut 41: 763-770.

2. Camacho, C. 1997. Trastornos gastroentéricos en cerdos. Rev. Cienc. Vet. 8: 8-10.

3. Davies, G.R.; M.E. Wilkie; D.S. Rampton. 1993. Effects of metronidazole and misoprostol on indomethacin-induced changes in intestinal permeability. Digest. Dis. Sci. 38: 417-425.

4. Davies, N.M.; F. Jamali. 1997. Pharmacological protection of NSAIDinduced intestinal permeability in the rat: effect of tempo and metronidazole as potential free radical scavengers. Hum. Exp. Toxicol. 16: 345-349.

5. Evans, S.M.; B.J. Whittle. 2001. Interactive roles of superoxide and induce nitric oxide synthase in rat intestinal injury provoked by non-steroidal antiinflammatory drugs. Eur. J. Pharmacol. 429: 287-296.

6. Gross, V.; H. Arndt; T. Andus; K. Palitzsch; J. Scholmerisch. 1994. Free radicals in inflammatory bowel disease pathophysiology and therapeutic implications. Hepato-gastroenterol. 41 : 320-327.

7. Milks, L.C.; G.P. Conyers; E.B. Cramer. 1986. The effect of neutrophil migration on epithelial permeability. J. Cell Biol. 103: 2729-2738.

8. Ramírez, A. 1990. Colibacilosis entérica en crías de alpacas En: Avances sobre investigación en salud animal. Camélidos sudamericanos. Bol. Div. IVITA 23: 20-21.

9. Ramos, D. 2003. Propuesta de un modelo experimental de enteritis aguda inducida por indometacina en ratas albinas. Tesis de Médico Veterinario. Facultad de Medicina Veterinaria, Univ. Nacional Mayor de San Marcos. Lima. 60 p.

10. Slauson, D.; B. Cooper. 1990. Mechanisms of disease. $2^{\text {nd }} e d . p 179$ 184. Ed. Williams \& Wilkins. USA.

11. Wax, J.; W.A. Clinger; P. Varner; P. Bass; C.V. Winder. 1970. Relationship of the enterohepatic cycle to ulcerogenesis in the rat small bowel with flufenamic acid. Gastroenterology 58: 772-780.

12. Yamada, T.; E. Deitch; R.D. Specian; M.A. Perry; R.B. Sartor; M.B. Grisham. 1993. Mechanisms of acute and chronic intestinal inflammation induced by indomethacin. Inflammation 17:641-662. 